\title{
DIFERENÇAS ENTRE AS DIMENSÕES DAS FIBRAS NOS ANEIS DE CRESCIMENTO DETERMINADOS NO D. A. P. E EM NÍVEIS DIFERENTES DO FUSTE DE ĀRVORES ADULTAS DE Eucaliptus Saligna SMITH *
}

\author{
Clóvis F. O. Santos \\ IzAIAS R. NogUeIra ***
}

\section{RESUMO}

\begin{abstract}
Neste trabalho apresentamos os resultados dos estudos das diferenças entre as dimensões (comprimento, diametro e espessura) das fibras lenhosas nos anéis de crescimento (da periferia ao centro do fuste), determinadas ao nivel do D. A. P. (distancia a altura do peito) e em diferentes niveis (10\%, $20 \%$ e $30 \%$ da altura do fuste) de árvores adultas de Eucalyptus saligna Smith, tendo como objetivo verificar se em nossas condiçб̃es, as amostras tomadas apenas no D. A. P. representam o fuste da árvore.
\end{abstract}

\section{INTRODUÇÃO}

A importância das características do comprimento e espessura das fibras, nas propriedades físicas e na qualidade da madeira é acentuada por MOTTET (1965), e ZOBEL (s/data).

O lenho adulto (formado no período de maturidade da árvore) possui características muito diferente das apresentadas pelo lenho juvenil (formado nos primeiros anos de vida da árvore), (ZOBEL, 1961).

Em trabalho inicial, constante de um plano de pesquisa sobre fibras de E. saligna Smith., mostrou SANTOS \& NOGUEIRA (1971) que para as condições de São Paulo a idade adulta do E. saligna avaliada pelas dimensões das fibras tomadas nos anéis de crescimento ao nível do D. A. P. é atingida entre $9 .^{\circ}$ e $10 .^{\circ}$ anel de crescimento.

\footnotetext{
- Entregue para publicação em 9/10/1974.

** Departamento de Botanica da ESALQ.

** Departamento de Matemática e Estatística da ESALQ.
} 
É fato conhecido que o número de anéis anuais de crescimento diminui da base em direção ao ápice do tronco, e que as variações nas dimensões das fibras em diferentes níveis do tronco segue o mesmo modelo de variação ao nível do D. A. P., bem como, varia da base para o ápice.

BISSET \& DADSWEL (1949) afirmam também que em uma determ1nada árvore o comprimento máximo das fibras do tronco de uma árvore atinge aproximadamente a $1 / 3$ da altura total do mesmo.

RUDMAN et al (1969) revelam que as amostras tomadas do tronco das árvores de três espécies de Eucalipłus ao nível do D. A. P. são inadequadas para o estudo qualitativo da madeira, principalmente quando se procura estabelecer e separar matrizes para trabalhos de melhoramento de essencias florestais.

Segundo esses autores, nos trabalhos de pesquisa sobre mensurações das dimensões das fibras deve-se levar em consideração a idade da árvore, altura do tronco e a posição no tronco onde a amostra é retirada.

Segundo RUDMAN et al (1969) concluiram que para três espécies de Eucaliptus estudadas para a seleção de matrizes, tomando como características o comprimento da fibra e a densidade há necessidade de se obter amostragem do tronco em posições mais altas do que o D. A. P.

Como no Brasil muitos trabaihos de pesquisa sobre fibras de Eucaliptus tem sido baseado em amostras tomadas apenas ao nível do D. A. P.; como nos trabalhos acima citados mostram a importância de se considerar para a escolha de árvores matrizes, a qualidade das fibras como seu elemento representativo, elaboramos a presente pesquisa com o objetivo de verificar se em nossas condições (Éstado de São Paulo) as amostras tomadas apenas ao nivel do D. A. P. representam o fuste todo da árvore.

\section{MATERIAL : MÉTODO}

Aproveitando o material do fuste das duas árvores empregadas para um trabalho anterior; (SAN'TOS \& NOGUEIRA, 1971), utilizamos neste trabalho das amostras em forma de cunha, retiradas de cada um dos discos da mesma face do fuste das duas árvores anteriormente empregadas, obtidas de três alturas diferentes do fuste, além do D. A. P., tais como: 10\%, 20\% e 30\% da altura do fuste, conforme preconiza RUDMAN et al (1969).

As dimensões das fibras tomadas nos três níveis diferentes do fuste foram comparadas com as dimensões no nível do D. A. P. obtidas no trabalho anterior, (SANTOS \& NOGUEIRA, 1971).

A técnica de masceração empregada foi a mesma já utilizada em trabalhos anteriores.

Pelo processo da micrometria empregando-se a ocular micrométrica, foram medidas ao acaso, o comprimento, o diâmetro externo e interno (que por diferenças dividida pela metade nos fornece a espessura das fibras) as quais em número de 5 , dissociadas e contida em cada lâmina, das qua- 
tro lâminas correspondentes a cada um dos anéis de crescimento, e nas alturas consideradas, das duas plantas analisadas (A e B).

Desse modo foram medidas 2.140 fibras a saber:

\begin{tabular}{|c|c|c|c|c|}
\hline \multirow{2}{*}{$\begin{array}{c}\text { Altura da } \\
\text { amostra no } \\
\text { tronco }\end{array}$} & \multicolumn{2}{|c|}{ Planta A } & \multicolumn{2}{c|}{ Planta B } \\
\cline { 2 - 5 } & N.0 de anéis & N.0 de fibras & N.0 de anéis & N.0 de fibras \\
\hline $10 \%$ & 21 & 420 & 21 & 420 \\
$20 \%$ & 18 & 360 & 17 & 340 \\
$30 \%$ & 15 & 300 & 15 & 300 \\
\hline
\end{tabular}

De cada fibra eram tomadas três dimensões diferentes a saber: 1) Comprimento (obtida com objetiva $6 \mathrm{x}$ ); 2) Diâmetro externo, e 3) Diâmetro interno (obtida com objetiva $45 \mathrm{x}$ ), totalizando 6.420 dimensões.

Para a realização das mensurações contamos com o auxílio dos alunos bolsistas junto ao Departamento de Botânica, Regina Frey Gonçalves, e Serafim Daniel Balestero, aos quais agradecemos.

Com o objetivo de estabelecer a correspondência dos anéis nas diferentes alturas (D. A. P. $10 \%, 20 \%$ e $30 \%$ ) os anéis foram numerados de fora para dentro do tronco, tendo em vista a atividade cambial ser constante e periódica em toda extensão do fuste durante a vida da árvore. Os anéis de crescimento mais externo são os recém-formados, e os mais internos são os formados no início da atividade cambial. (Ver figura 1).

Foram feitas a análise de variância, aplicando-se 0 teste $F$ e o teste Tukey para as comparações das médias dos comprimentos, dos diâmetros externos e das espessuras das fibras nos diferentes níveis das duas plantas estudadas.

Além da análise da variância foi feita também a determinação das equações de regressão dos valores médios (comprimento, diâmetro externo e espessura das fibras) em relação a ordem dos anéis, do primeiro ao décimo quinto, seguindo na ordem da periferia para o centro.

\section{RESULTADOS OBTIDOS}

Pela contagem do número dos anéis de crescimento do fuste das plantas A e B foi verificado que na altura de $10 \%$ do tronco de ambas, ocorria 21 anéis de crescimento (mesmo número do que ao nível do $\mathrm{D}$. A. P.) (SANTOS \& NOGUEIRA, 1971) e nas alturas de $20 \%$ e $30 \%$ ocorria 18 e 15 
anéis respectivamente, para a planta $A$; porém na planta $B$ nas alturas de $20 \%$ e $30 \%$ ocorria 17 e 15 anéis respectivamente.

Desse modo tanto para planta A, como para planta B, só puderam ser comprovados os resultados das médias do comprimento, do diâmetro externo e da espessura das fibras, de somente 15 anéis de crescimento do primeiro (anel mais externo) ao $15^{\circ}$ anel (mais interno).

Os resultados das médias das mensurações microscópicas das fibras dos 15 anéis comparáveis das plantas (A e B) constam do quadro 1 e 2 .

A análise da variância dos resultados médios das dimensões das duas plantas nas três alturas (10\%, $20 \%$ e $30 \%$ da altura do fuste) nos mostrou que para a planta $\mathrm{A}$ elas não diferem entre si quando comparadas duas a duas, porém o D.A.P difere das outras alturas ao nível de $1 \%$ de probabilidade, para as médias do comprimento e diâmetro externo das fibras, e 5\% para a espessura das mesmas.

Para a planta B a análise revelou aspectos semelhantes, isto é, o D.A.P difere das outras alturas ao nível de $1 \%$ de probabilidade para as médias do comprimento e espessura das fibras, e $5 \%$ de probabilidade para as médias do seu diâmetro externo.

Os quadros de 3 a 14 mostram os valores médios em micros e as respectivas análises de variância.

\section{DISCUSSÃO}

Fazendo-se uma análise do gráfico dos comprimentos das fibras (gráficos $n^{\circ} \mathrm{s} 1$ e 3 ) ou dos seus valores médios (quadros 3 e 9) pode-se verificar que em dado anel a medida do comprimento das fibras aumenta com a altura do fuste, pois, para as alturas houve uma variação significativa ao nível de $1 \%$ de probabilidade. Isso nos mostra que em qualquer dos anéis de crescimento, a média do comprimento das fibras cresce um pouco até o nível de $10 \%$ do fuste, e depois fica mais ou menos estável, ou decresce aos níveis de 20 a $30 \%$.

Em quaisquer dos níveis do D.A.P, 10,20 e $30 \%$, o comprimento das fibras parecem seguir um mesmo modêlo de crescimento do mais interno ao mais externo, isto é, sempre mais curtos nos anéis mais internos e vão aumentando quanto mais próximo da periferia.

Para o diâmetro externo e espessura observa-se pelos valores médios (quadros $5,7,11$ e 13, e gráficos nos 2 e 4) que em um determinado anel o diâmetro e espessura das fibras decrescem com a altura e que quaisquer dos níveis do D.A.P, $10 \%, 20 \%$ e $30 \%$ os valores médios são sempre menores nos anéis mais internos.

Essas observações concordam em parte com os estudos em E. globulus feito por CARVALHO (1962).

Com os dados obtidos constantes dos quadros de 3 a 14 e suas análises de variância foi possível a construção dos gráficos 1 a 4, bem como, 
empregar a seguinte equação de regressão, obtida dos valores médios dos comprimentos, diâmetro externo e espessura das fibras em relação com os anéis de crescimento:

$$
\begin{aligned}
& \hat{Y}=\hat{a}+\hat{b} x_{i}, \text { onde } \\
& \hat{Y}_{i}=\hat{A}=\text { média do anel }\left(x_{i}=1,2, \ldots \ldots, 15\right)
\end{aligned}
$$

Pelo traçado da reta das duas plantas A e B calculado pela equação da regressão acima referida, pode-se verificar que a média dos comprimentos das fibras nas alturas de $10 \%, 20 \%$ e $30 \%$ nas duas plantas estão em torno da reta de regressão e que a média do D.A.P está bem mais distante e abaixo da reta.

Para a média das espessuras, verifica-se pelo gráfico e pelos valores calculados pela equação da regressão, que os valores médios do D.A.P. ficam por cima da reta determinada pela referida equação, e que as médias observadas ao nível de $10 \%, 20 \%$ e $30 \%$ ficam abaixo da mesma, numa posição inversa portanto dos resultados médios dos comprimentos.

Comparando-se os gráficos ou as médias dos comprimentos com as médias dos diâmetros e das espessuras de ambas plantas (A e B), observa-se que num dado anel e ao nível do D.A.P a média dos comprimentos das fibras é menor que a média ao nível 10\%, $20 \%$ e $30 \%$. Entretanto, para as médias das espessuras os resultados se invertem.

Dessa maneira, pode-se afirmar que as fibras dentro do mesmo anel e ao longo do tronco aumentam o seu comprimento aos níveis de $10 \%$, $20 \%$ e $30 \%$ do fuste, porém, a sua espessura e diâmetro diminui. Esse fato não é referido pelos autores consultados na literatura.

E fato biologicamente comprovado que quando o ritmo de crescimento de um vegetal é mais ou menos estável, o aumento de comprimento de suas células, fibras, vasos e etc., é compensado pela diminuição no seu diâmetro e espessura, indicando que o teor de matéria seca (celulose, lignina e etc.,) produzidas são mais ou menos constante.

\section{CONCLUSÓES}

$1^{0}$ - Há uma diferença significativa entre os valores médios tomadas ao nível do D.A.P e aqueles tomados ao nível de $10 \%, 20 \%$ e $30 \%$ da altura do fuste.

$2^{\circ}$ - Em um dado anel e ao longo do fuste, o comprimento médio das fibras aumentam do D.A.P para os níveis de $10 \%, 20 \%$ e $30 \%$ de altura do mesmo, enquanto o diâmetro e espessura da fibra diminui.

$3^{0}$ - Os resultados por nós analisados vem confirmar as afirmações de BISSET \& DASDWELL (1949), em que afirmam que o máximo com- 
primento médio das fibras atinge aproximaảamente ao terço superior da altura do fuste das árvores; também concorda com RUDMAN et al (1969), os quais afirmam que as amostras tomadas ao nível do D.A.P são inadequadas para estudos qualitativos da madeira, principalmente para os trabalhos de seleção de matrizes e de melhoramentos das essências florestais.

\section{SUMMARY}

VARIATION OF FIBER DIMENTIONS IN GROWTH RINGS, TAKEN AT BREAST HEIGHT AND AT THREE ADITIONAL LEVEL OF TRUNK HEIGHT.

This paper deals with the variation of fiber dimentions in growth rings taken at the breast height (B.H.) and at three aditional level of trunk height $(10 \%, 20 \%$ and $30 \%$ of total height) of two plants of Eucalyptus saligna Smith.

Statistical analyses of variance for fiber length, fiber diameter and fiber thikness in diferent growth rings at B.H., 10\%, 20\% and $30 \%$ of trunk height is prsented.

Analysis of variance allowed the following conclusion:

$1^{0}$ - Significant diferences were observed for fiber dimentions mean, taken at B.H. and those taken at the levels of $10 \%, 20 \%$ and $30 \%$ of trink height;

$2^{0}$ - In any growth rings along the trunk, the fiber length means increased consistently from B.H. to the levels of $10 \%, 20 \%$ and $30 \%$ of trink height, however the means for fiber diameter and thikness of the wall decreace from the B.H. point to the leves of $10 \%, 20 \%$ and $30 \%$ of trink height;

$3^{0}$ - The results obtained in the present study confirm those obtained by BISSET \& DASDWELL (1949) in that the maximum fiber lenght is reached at aproxmately one third of the total height of the trink and also confirm the afirmation made by RUDMAN et al (1969) that the samples taken at the B.H. level are inadequate for qualitative wood studies, specially in raltion with forest genetics investigation.

\section{LITERATURA CITADA}

BISSET, L. J., and DASDWELL, H. E. - 1949 - The variation of fiber length within one tree of Eucalyptus regnan. Australian Forestry 13 (12) : 86-96.

CARvalHo, A. 1962 - Madeira de Eucalipto. Lisboa, Est. e Div. Tec. Serviços Florestais. 62 pgs.

MOTTET, A. 1965 - Considerations on the relationship betwen certain anatomical characteristics and density in tropical woods. Proceedings IUFRO - Section 41. 2 : $1-14$. 
RUDMAN, P.; HIGGS, M.; DAVIDSON, J.; and MALAJCZURK, N. $1969-$ Breeding eucalyptus for wood properties. In: WORLD CONSULTATION ON FOREST TREE BREEDING, 2., Washington. Voluntary paper. Rome, F.A.O/ IUFRO, Sect. II, p. 1-9.

SANTOS, Clóvis Ferraz de Oliveira \& NOGUEIRA, Isaias Rangel. 1971 - A idade adulta do Eucalyptus saligna Smith, em Rio Claro, Estado de São Paulo, determinada pelas dimensões das fibras. Anais da Escola Superior de Agricultura «Luiz de Queiroz», Piracicaba, 28 : 165-175.

ZOBEL, B., - (s/ data) - Wood quality improvement through better trees. Raleigh, North Carolina State University, School of Forestry, 6 pgs.

ZOBEL, B., 1961 - Juvenility in Wood Production. (Problems in forest tree Breeding). Recent advances in Botany. 2 : 1663-1665. 
Quadro I: Médias das mensurações microscópicas das fibras de 15 anéis comparáveis (Planta A)

\begin{tabular}{|c|c|c|c|c|c|c|c|c|c|c|c|c|}
\hline \multirow{2}{*}{$\begin{array}{l}\text { Námero } \\
\text { dos } \\
\text { Anéis }\end{array}$} & \multicolumn{3}{|c|}{ D.A.P. } & \multicolumn{3}{|c|}{$10 \%$} & \multicolumn{3}{|c|}{$20 \%$} & \multicolumn{3}{|c|}{$30 \%$} \\
\hline & Comp. & $\begin{array}{l}\text { Diam. } \\
\text { Ext. }\end{array}$ & Espes. & Comp. & $\begin{array}{l}\text { Diam. } \\
\text { Ext. }\end{array}$ & Espes. & Comp. & $\begin{array}{l}\text { Diam } \\
\text { Ext. }\end{array}$ & Espes & Comp & $\begin{array}{l}\text { Diam } \\
\text { Ext. }\end{array}$ & Espes. \\
\hline $1.0+$ ext. & 10,04 & 1,61 & 0,464 & 12,27 & 1,45 & 0,474 & 11,47 & 1,72 & 0,413 & 12,52 & 1,52 & 0,355 \\
\hline 2.0 & 11,26 & 1,48 & 0,425 & 12,16 & 1,32 & 0,382 & 12,42 & 1,69 & 0,386 & 12,78 & 1,58 & 0,341 \\
\hline 3.0 & 11,68 & 1,44 & 0,399 & 13,09 & 1,48 & 0,407 & 12,35 & 1,54 & 0,439 & 12,42 & 1,56 & 0,354 \\
\hline 4.0 & 10,80 & 1,57 & 0,378 & 12,61 & 1,52 & 0,408 & 11,92 & 1,57 & 0,437 & 12,24 & 1,60 & 0,350 \\
\hline 5.0 & 11,16 & 1,71 & 0,439 & 12,77 & 1,54 & 0,383 & 11,93 & 1,36 & 0,395 & 12,33 & 1,44 & 0,338 \\
\hline 6.0 & 11,34 & 1,55 & 0,512 & 12,58 & 1,31 & 0,399 & 12,12 & 1,44 & 0,434 & 12,49 & 1,39 & 0,334 \\
\hline 7.0 & 12,00 & 1,88 & 0,535 & 12,45 & 1,32 & 0,395 & 12,23 & 1,35 & 0,437 & 12,10 & 1,39 & 0,340 \\
\hline 8.0 & 11,31 & 1,75 & 0,457 & $\mid 13,14$ & 1,44 & 0,385 & 12,79 & 1,39 & 0,420 & 11,33 & 1,32 & 0,335 \\
\hline 9.0 & 10,62 & 1,73 & 0,453 & 12,82 & 1,43 & 0,364 & 12,55 & 1,29 & 0,343 & 11,81 & 1,41 & 0,384 \\
\hline 10.0 & 12,22 & 1,70 & 0,432 & 12,90 & 1,45 & 0,357 & 12,18 & 1,36 & 0,392 & 11,97 & 1,31 & 0,341 \\
\hline 11.0 & 11,04 & 1,81 & 0,461 & 12,99 & 1,35 & 0,342 & 11,83 & 1,30 & 0,339 & 11,34 & 1,56 & 0,354 \\
\hline 12.0 & 11,90 & 1,61 & 0,423 & 12,09 & 1,46 & 0,323 & 11,62 & 1,43 & 0,376 & 11,40 & 1,52 & 0,389 \\
\hline 13.0 & 11,39 & 1,69 & 0,416 & 11,27 & 1,48 & 0,320 & 12,38 & 1,50 & 0,336 & 10,87 & 1,47 & 0,298 \\
\hline 14.0 & 10,67 & 1,61 & 0,372 & 10,88 & 1,39 & 0,310 & 11,50 & 1,27 & 0,331 & 10,89 & 1,46 & 0,302 \\
\hline 15.0 & 10,66 & 1,57 & 0,369 & 11,10 & 1,48 & 0,311 & 11,51 & 1,22 & 0,250 & 10,10 & 1,42 & 0,328 \\
\hline
\end{tabular}

OBS.: Média dos comprimentos obj. $6 \times$

Média dos diâmetros externos e espessura obj. $45 \times$

Quadro II: Médias das mensurações microscópicas das fibras de 15 anéis comparáveis (Planta B)

\begin{tabular}{|c|c|c|c|c|c|c|c|c|c|c|c|c|}
\hline \multirow{2}{*}{$\begin{array}{l}\text { Número } \\
\text { dos } \\
\text { Anéis }\end{array}$} & \multicolumn{3}{|c|}{ D.A.P. } & \multicolumn{3}{|r|}{$10 \%$} & \multicolumn{3}{|r|}{$20 \%$} & \multicolumn{3}{|r|}{$30 \%$} \\
\hline & Comp. & $\begin{array}{l}\text { Diam. } \\
\text { Ext. }\end{array}$ & Esp. & Comp. & $\begin{array}{l}\text { Diam. } \\
\text { Ext. }\end{array}$ & Espes. & Comp. & Diam. & Espes. & Comp. & $\begin{array}{l}\text { Diam. } \\
\text { Ext. }\end{array}$ & Espes. \\
\hline $1.0+$ ext. & 10,40 & 1,76 & 0,485 & 12,58 & 1,47 & 0,322 & 12,18 & 1,62 & 0,340 & 12,78 & 1,50 & 0,322 \\
\hline 2.0 & 11,41 & 1,53 & 0,477 & 12,99 & 1,49 & 0,331 & 12,98 & 1,58 & 0,358 & 12,98 & 1,60 & 0,339 \\
\hline 3.0 & 11,39 & 1,41 & 0,325 & 13,61 & 1,58 & 0,345 & 12,75 & 1,64 & 0,341 & 12,47 & 1,50 & 0,311 \\
\hline $4 .{ }^{\circ}$ & 10,35 & 1,58 & 0,381 & 13,24 & 1,47 & 0,331 & 12,92 & 1,49 & 0,347 & 12,53 & 1,55 & 0,326 \\
\hline 5.0 & 11,27 & 1,74 & 0,437 & 12,86 & 1,52 & 0,335 & 12,16 & 1,57 & 0,350 & 12,58 & 1,46 & 0,338 \\
\hline 6.0 & 11,67 & 1,55 & 0,421 & 13,61 & 1,60 & 0,339 & 13,32 & 1,53 & 0,343 & 13,49 & 1,47 & 0,327 \\
\hline 7.0 & 11,65 & 1,76 & 0,599 & 12,65 & 1,52 & 0,345 & 12,25 & 1,56 & 0,337 & 12,15 & 1,49 & 0,335 \\
\hline 8.0 & 11,51 & 1,68 & 0,503 & 13,74 & 1,58 & 0,343 & 12,79 & 1,62 & 0,326 & 12,18 & 1,62 & 0,341 \\
\hline 9.0 & 10,75 & 1,67 & 0,546 & 12,92 & 1,56 & 0,338 & 12,02 & 1,50 & 0,336 & 12,48 & 1,53 & 0,326 \\
\hline 10.0 & 11,22 & 1,79 & 0,468 & 12,84 & 1,48 & 0,319 & 12,72 & 1,51 & 0,335 & 12,68 & 1,50 & 0,347 \\
\hline 11.0 & 11,32 & 1,78 & 0,525 & 13,08 & 1,47 & 0,334 & 12,02 & 1,61 & 0,351 & 12,34 & 1,44 & 0,335 \\
\hline 12.0 & 11,63 & 1,64 & 0,464 & 13,10 & 1,54 & 0,328 & 11,91 & 1,47 & 0,340 & 11,80 & 1,41 & 0,319 \\
\hline 13.0 & 11,06 & 1,71 & 0,425 & 11,27 & 1,47 & 0,326 & 12,48 & 1,50 & 0,350 & 11,07 & 1,40 & 0,322 \\
\hline $14 .{ }^{\circ}$ & 10,28 & 1,59 & 0,389 & 11,05 & 1,54 & 0,326 & 10,59 & 1,64 & 0,329 & 11,08 & 1,54 & 0,331 \\
\hline $15 .{ }^{\circ}$ & 10,54 & 1,52 & 0,382 & 11,23 & 1,42 & 0,305 & 11,53 & 1,54 & 0,334 & 10,02 & 1,54 & 0,302 \\
\hline
\end{tabular}

OBS.: Média dos comprimentos obj. $6 \times$

Média dos diâmetros externos e espessura obj. $45 \times$ 
Quadro III: Valores médios em micros do comprimento das fibras nos anéis de crescimento em diferentes níveis. (Planta A).

\begin{tabular}{l|c|c|c|c|c|c}
\hline \multirow{2}{*}{$\begin{array}{l}\text { Anéis de crescimen } \\
\text { to da casca para } \\
\text { dentro }\end{array}$} & \multicolumn{3}{|c|}{ Média dos Comprimentos das Fibras } & \multicolumn{2}{c}{ Valores } \\
\cline { 2 - 7 } & D.A.P. & $10 \%$ & $20 \%$ & $30 \%$ & $\begin{array}{c}\text { Calculados } \\
\text { em micros }\end{array}$ & $\begin{array}{c}\text { Observados } \\
\text { em micros }\end{array}$ \\
\hline $1.0+$ ext. & $1.074,28$ & $1.312,89$ & $1.227,29$ & $1.339,64$ & $1.313,96$ & $1.239,06$ \\
2.0 & $1.204,82$ & $1.301,12$ & $1.328,94$ & $1.367,46$ & $1.307,54$ & $1.301,12$ \\
3.0 & $1.249,76$ & $1.400,63$ & $1.321,45$ & $1.328,94$ & 1.300 .05 & $1.325,73$ \\
4.0 & $1.155,60$ & $1.349,27$ & $1.275,44$ & $1.309,68$ & $1.292,56$ & $1.272,23$ \\
5.0 & $1.194,12$ & $1.366,39$ & $1.276,51$ & $1.319,31$ & $1.286,14$ & $1.289,35$ \\
6.0 & $1.213,38$ & $1.346,06$ & $1.296,84$ & $1.336,43$ & $1.278,75$ & $1.297,91$ \\
7.0 & $1.284,00$ & $1.332,15$ & $1.308,61$ & $1.294,70$ & $1.271,16$ & 1.305 .40 \\
8.0 & $1.210,17$ & $1.405,98$ & $1.357,83$ & $1.212,31$ & $1.264,74$ & $1.296,84$ \\
9.0 & $1.136,34$ & $1.371,74$ & $1.342,85$ & $1.263,27$ & $1.257,25$ & $1.278,65$ \\
10.0 & $1.200,54$ & $1.304,33$ & $1.303,26$ & $1.280,79$ & $1.249,76$ & $1.272,23$ \\
11.0 & $1.181,28$ & $1.389,93$ & $1.265,81$ & $1.213,38$ & $1.242,27$ & $1.185,56$ \\
12.0 & $1.273,30$ & $1.304,33$ & $1.243,34$ & $1.219,80$ & $1.235,85$ & $1.260,46$ \\
13.0 & $1.218,73$ & $1.205,89$ & $1.324,66$ & $1.173,09$ & $1.228,36$ & $1.228,36$ \\
14.0 & $1.141,69$ & $1.164,16$ & $1.230,50$ & $1.165,23$ & $1.220,87$ & $1.175,93$ \\
15.0 + int.) & $1.138,48$ & $1.187,70$ & $1.231,57$ & $1.080,70$ & $1.214,45$ & $1.159,88$ \\
\hline Média & $1.191,98$ & $1.316,10$ & $1.289,35$ & $1.259,39$ & & \\
\hline
\end{tabular}

Quadro IV: Análise da variância das meédias dos comprimentos das fibras nos diferentes níveis. (Planta A)

\begin{tabular}{|l|c|c|c|c|}
\hline & G.L. & S.Q. & Q.M. & F \\
\hline Altura & 3 & 11,26 & 3,75 & $15,00 * *$ \\
\hline Anéis & 14 & 10,73 & 0,77 & $3,08 * *$ \\
\hline Resíduo & 42 & 10,50 & 0,25 & \\
\hline
\end{tabular}

1) - Para as alturas houve uma variação significativa ao nível de $1 \%$ de probabilidade.

2) - O D. A. P. difere das outras alturas ao nível de $1 \%$ de probabilidade.

3) - Houve uma diferença significativa ao nível de $1 \%$ para os aneis.

OBS.: os dados desta análise não são em micros. 
Quadro V: Valores médios em micros do diâmetro externo das fibras nos anéis de crescimento em diferentes niveis (Planta A)

\begin{tabular}{l|c|c|c|c|c|c}
\hline $\begin{array}{l}\text { Aneis de crescimen- } \\
\text { to da casca para } \\
\text { dentro }\end{array}$ & \multicolumn{3}{|c|}{ Média dos Diâmetros Externos das Fibras } & \multicolumn{2}{c}{ Valores } \\
\cline { 2 - 7 } & D.A.P. & $10 \%$ & $20 \%$ & $30 \%$ & $\begin{array}{l}\text { Calculados } \\
\text { em micros }\end{array}$ & $\begin{array}{l}\text { Observados } \\
\text { em micros }\end{array}$ \\
\hline 1.0 (+ ext.) & 20,93 & 19,24 & 22,36 & 19,76 & 20,02 & 20,54 \\
2.0 & 19,24 & 17,16 & 21,97 & 20,54 & 19,89 & 19,76 \\
3.0 & 18.72 & 19,24 & 20,02 & 20,28 & 19,56 & 19,63 \\
4.0 & 20,41 & 19,76 & 20,41 & 20,80 & 19,76 & 20,41 \\
5.0 & 22,23 & 20,02 & 17,68 & 18,72 & 19,63 & 19,63 \\
6.0 & 20,15 & 17,03 & 18,72 & 18,07 & 19,63 & 18,46 \\
7.0 & 24,44 & 17,16 & 17,55 & 18,07 & 13,65 & 19,37 \\
8.0 & 22,75 & 18,72 & 18,07 & 17,16 & 19,37 & 19,24 \\
9.0 & 22,49 & 18,51 & 16,77 & 18,33 & 19,37 & 19,11 \\
10.0 & 22,10 & 18,85 & 17,68 & 17,03 & 19,24 & 18,98 \\
11.0 & 23,53 & 17,55 & 16,90 & 20,28 & 19,24 & 19,63 \\
12.0 & 20,93 & 18,98 & 18,59 & 19,76 & 19,11 & 19,63 \\
13.0 & 21,97 & 19,24 & 19,50 & 19,11 & 18,98 & 20,02 \\
14.0 & 20,93 & 18,07 & 16,51 & 18,98 & 18,98 & 18,59 \\
15.0 (+ int.) & 20,41 & 18,85 & 15,86 & 18,46 & 18,85 & 18,46 \\
\hline Média & 21,41 & 18,56 & 18,57 & 19,02 & & \\
\hline
\end{tabular}

Quadro VI: Análise da variância das médias dos diâmetros externos das fibras nos diferentes niveis. (Planta A)

\begin{tabular}{|c|c|c|c|}
\hline G.L. & S.Q. & O.M. & F \\
\hline 14 & 0,126 & 0,009 & 0,64 \\
\hline 3 & 0,496 & 0,165 & $11,79 * * *$ \\
\hline 42 & 0,584 & 0,014 & \\
\hline
\end{tabular}

D. A. P. difere das demais alturas ao nível de $1 \%$ de probabilidade. 
Quadro VII: Valores médios em micros da espessura das fibras nos anéis de crescimento em diferentes niveis. (Planta A)

\begin{tabular}{l|l|c|c|c|c|c}
\hline $\begin{array}{l}\text { An6is de crescimen- } \\
\text { to da casca para } \\
\text { dentro }\end{array}$ & \multicolumn{3}{|c|}{ Média das espessuras das fibras } & \multicolumn{2}{c}{ Valores } \\
\cline { 2 - 6 } & D.A.P. & $10 \%$ & $20 \%$ & $30 \%$ & $\begin{array}{c}\text { Calculados } \\
\text { em micros }\end{array}$ & $\begin{array}{c}\text { Observados } \\
\text { em micros }\end{array}$ \\
\hline 1.0 (+ ext.) & 6,032 & 6,162 & 5,369 & 4,628 & 5,590 & 5,551 \\
2.0 & 5,525 & 4,966 & 5,018 & 4,433 & 5,434 & 4,992 \\
3.0 & 5,187 & 5,291 & 5,707 & 4,602 & 5,434 & 5,200 \\
4.0 & 4,914 & 5,304 & 5,681 & 4,550 & 5,278 & 5,109 \\
5.0 & 5,707 & 4,979 & 5,135 & 4,394 & 5,200 & 5,057 \\
6.0 & 6,656 & 5,187 & 5,642 & 4,342 & 5,122 & 5,460 \\
7.0 & 6,955 & 5,135 & 5,681 & 4,420 & 5,044 & 5,551 \\
8.0 & 5,941 & 5,005 & 5,460 & 4,355 & 4,966 & 5,187 \\
9.0 & 5,889 & 4,732 & 4,459 & 4,992 & 4,888 & 5,018 \\
10.0 & 5,616 & 4,641 & 5,096 & 4,433 & 4,810 & 4,953 \\
11.0 & 5,993 & 4,446 & 4,407 & 4,602 & 4,732 & 4,862 \\
12.0 & 5,499 & 4,199 & 4,368 & 5,057 & 4,654 & 4,784 \\
13.0 & 5,408 & 4,160 & 4,368 & 3,874 & 4,576 & 4,459 \\
14.0 & 4,836 & 4,030 & 4,303 & 3,926 & 4,498 & 4,277 \\
15.0 (+ int.) & 4,797 & 4,043 & 3,250 & 4,624 & 4,420 & 4,095 \\
\hline Média & 5,664 & 4,819 & 4,930 & 4,482 & & \\
\hline
\end{tabular}

Quadro VIII: Análise da variância das médias das espessuras das fibras nos diferentes níveis. (Planta A)

\begin{tabular}{|l|r|l|l|l|}
\hline & G.L. & S.Q. & \multicolumn{1}{|c|}{ Q.M. } & \multicolumn{1}{|c|}{ F } \\
\cline { 2 - 5 } Anéis & 14 & 0,06182 & 0,004416 & $3,79^{*}$ \\
Altura & 3 & 0,06814 & 0,02271 & $19,50^{* *}$ \\
Resíduo & 42 & 0,048914 & 0,001165 & \\
\hline
\end{tabular}

Há efeito significativo de aneis ao nível de $5 \%$ e de alturas ao nível de $1 \%$. D. A. P. difere das demais alturas ao nível de $1 \%$ de probabilidade. 
Quadro IX: Valores médios em micros do comprimento das fibras nos anéis de crescimento am diferentes niveis (Planta $B$ )

\begin{tabular}{l|c|c|c|c|c|c}
\hline \multirow{2}{*}{$\begin{array}{l}\text { Anéis de crescimen- } \\
\text { to da casca para } \\
\text { dentro }\end{array}$} & \multicolumn{3}{|c|}{ Média dos comprimentos das fibras } & \multicolumn{2}{c}{ Valores } \\
\cline { 2 - 7 } & D.A.P. & $10 \%$ & $20 \%$ & $30 \%$ & $\begin{array}{c}\text { Calculados } \\
\text { em micros }\end{array}$ & $\begin{array}{c}\text { Observados } \\
\text { em micros }\end{array}$ \\
\hline 1.0 (+ ext.) & $1.112,80$ & $1.346,06$ & $1.303,26$ & $1.367,46$ & $1.366,39$ & $1.282,93$ \\
2.0 & $1.220,87$ & $1.389,93$ & $1.388,86$ & $1.388,86$ & $1.355,69$ & $1.347,13$ \\
3.0 & $1.218,73$ & $1.456,27$ & $1.364,25$ & $1.334,29$ & 1.343 .92 & $1.343,92$ \\
4.0 & $1.107,45$ & $1.416,68$ & $1.382,44$ & $1.340,71$ & $1.333,22$ & $1.311,82$ \\
5.0 & $1.205,89$ & $1.376,02$ & $1.301,12$ & $1.346,06$ & $1.322,52$ & $1.307,54$ \\
6.0 & $1.248,69$ & $1.456,27$ & $1.425,24$ & $1.443,43$ & $1.311,82$ & $1.393,14$ \\
7.0 & $1.246,55$ & $1.353,55$ & $1.310,75$ & $1.300,05$ & $1.301,12$ & $1.303,26$ \\
8.0 & $1.231,57$ & $1.470,18$ & $1.368,53$ & $1.303,26$ & $1.290,42$ & $1.343,92$ \\
9.0 & $1.150,25$ & $1.382,44$ & $1.286,14$ & $1.335,36$ & $1.279,72$ & $1.288,28$ \\
10.0 & $1.200,54$ & $1.373,88$ & $1.361,04$ & $1.356,76$ & $1.269,02$ & $1.323,58$ \\
11.0 & $1.211,24$ & $1.399,56$ & $1.286,14$ & $1.320,38$ & $1.258,32$ & $1.304,33$ \\
12.0 & $1.244,41$ & $1.401,70$ & $1.274,37$ & $1.262,60$ & $1.247,62$ & $1.295,77$ \\
13.0 & $1.183,42$ & $1.205,88$ & $1.335,36$ & $1.184,49$ & $1.235,85$ & $1.227,29$ \\
14.0 & $1.099,96$ & $1.182,35$ & $1.133,13$ & $1.089,25$ & $1.225,15$ & $1.123,50$ \\
15.0 (+ int.) & $1.127,78$ & $1.201,61$ & $1.233,71$ & $1.072,14$ & $1.214,45$ & $1.158,81$ \\
\hline Média & $1.187,70$ & $1.361,04$ & $1.317,17$ & $1.295,77$ & & \\
\hline
\end{tabular}

Quadro X: Análise da variância das médias do comprimento das fibras nos diferentes níveis. (Planta B)

\begin{tabular}{|l|r|r|r|c|}
\hline & G.L. & S.Q. & Q.M. & F \\
\cline { 2 - 5 } Anéis & 14 & 24,7531 & 1,7681 & $7,8722^{*}$ \\
Altura & 3 & 21,3724 & 7,1241 & $31,7190^{*} *$ \\
Resíduo & 42 & 9,4345 & 0,2246 & \\
\hline
\end{tabular}

Só o D. P. A. difere das outras alturas ao nível de $1 \%$ de probabilidade. 
Quadro XI: Valores médios em micros da espessura das fibras nos anéis de crescimento em diferentes niveis. (Planta B).

\begin{tabular}{l|l|l|l|l|l|l}
\hline $\begin{array}{l}\text { Anéis de crescimen- } \\
\text { to da casca para } \\
\text { dentro }\end{array}$ & \multicolumn{3}{|c|}{ Média das ospessuras das fibras } & \multicolumn{2}{c}{ Valores } \\
\cline { 2 - 7 } & D.A.P. & $10 \%$ & $20 \%$ & $30 \%$ & $\begin{array}{c}\text { Calculados } \\
\text { om micros }\end{array}$ & $\begin{array}{l}\text { Observados } \\
\text { om micros }\end{array}$ \\
\hline $1.0(+$ ext.) & 6,305 & 4,316 & 4,420 & 4,186 & 4,849 & 4,807 \\
2.0 & 6,201 & 4,303 & 4,654 & 4,407 & 4,836 & 4,891 \\
3.0 & 4,225 & 4,485 & 4,433 & 4,043 & 4,810 & 4,296 \\
4.0 & 4,953 & 4,303 & 4,511 & 4,238 & 4,797 & 4,501 \\
5.0 & 5,681 & 4,355 & 4,550 & 4,394 & 4,771 & 4,745 \\
6.0 & 5,473 & 4,407 & 4,459 & 4,251 & 4,745 & 4,647 \\
7.0 & 7,787 & 4,485 & 4,381 & 4,355 & 4,732 & 5,252 \\
8.0 & 6,539 & 4,459 & 4,238 & 4,333 & 4,706 & 4,917 \\
9.0 & 7,098 & 4,394 & 4,368 & 4,238 & 4,680 & 5,024 \\
10.0 & 6,084 & 4,147 & 4,355 & 4,511 & 4,667 & 4,774 \\
11.0 & 6,825 & 4,342 & 4,563 & 4,355 & 4,641 & 5,021 \\
12.0 & 6,032 & 4,264 & 4,420 & 4,147 & 4,615 & 4,716 \\
13.0 & 5,525 & 4,238 & 4,550 & 4,186 & 4,602 & 4,625 \\
14.0 & 5,057 & 4,238 & 4,277 & 4,303 & 4,576 & 4,469 \\
15.0 (+ int.) & 4,966 & 3,965 & 4,342 & 3,926 & 4,550 & 4,300 \\
\hline Média & 5,808 & 4,299 & 4,426 & 4,247 & & \\
\hline
\end{tabular}

Quadro XII: Análise da variância das médias da espessura das fibras nos diferentes níveis.(Planta B).

\begin{tabular}{|l|r|c|l|c|}
\hline & G.L. & S.Q. & QM. & F \\
\cline { 2 - 5 } Anéis & 15 & 0,03229 & 0,00215 & 1,547 \\
Alturas & 3 & 0,15800 & 0,5270 & $37,99^{*} *$ \\
Residuo & 45 & 0,06236 & 0,00139 & \\
\hline
\end{tabular}

Os aneis não diferem significativamente.

As alturas diferem significativamente ao nível de $1 \%$ de probabilidade. 
Quadro XIII: Valores médios em micros do diâmetro externo das fibras nos anéis de crescimento em diferentes niveis. (Planta B).

\begin{tabular}{l|l|c|c|c|c|c}
\hline \multirow{2}{*}{$\begin{array}{l}\text { Antis de crescimen- } \\
\text { to da casca para } \\
\text { dentro }\end{array}$} & \multicolumn{3}{|c|}{ Módia dos diâmetros externos das fibras } & \multicolumn{2}{c}{ Valores } \\
\cline { 2 - 7 } & D.A.P. & $10 \%$ & $20 \%$ & $30 \%$ & $\begin{array}{c}\text { Calculados } \\
\text { om micros }\end{array}$ & $\begin{array}{c}\text { Obsorvados } \\
\text { om micros }\end{array}$ \\
\hline 1.0 (+ oxt.) & 22,88 & 19,11 & 21,06 & 19,50 & 20,45 & 20,64 \\
2.0 & 19,89 & 19,37 & 20,54 & 20,80 & 20,41 & 20,15 \\
3.0 & 18,33 & 20,54 & 21,32 & 19,50 & 20,37 & 19,92 \\
4.0 & 20,54 & 19,11 & 19,37 & 20,15 & 20,33 & 19,79 \\
5.0 & 22,62 & 19,76 & 20,41 & 18,92 & 20,29 & 20,44 \\
6.0 & 20,15 & 20,80 & 19,89 & 19,11 & 20,25 & 19,99 \\
7.0 & 22,88 & 19,76 & 20,28 & 19,37 & 20,23 & 20,70 \\
8.0 & 21,84 & 20,54 & 21,06 & 21,06 & 20,19 & 21,12 \\
9.0 & 21,71 & 20,28 & 19,50 & 19,89 & 20,15 & 20,34 \\
10.0 & 23,27 & 19,24 & 19,63 & 19,50 & 20,11 & 20,41 \\
11.0 & 23,14 & 19,11 & 20,93 & 18,72 & 20,07 & 20,47 \\
12.0 & 21,32 & 20,02 & 19,11 & 18,33 & 20,05 & 19,69 \\
13.0 & 22,23 & 19,11 & 19,50 & 18,20 & 20,01 & 19,76 \\
14.0 & 20,67 & 20,02 & 21,32 & 20,02 & 19,97 & 20,51 \\
15.0 (+ int.) & 19,76 & 18,46 & 20,02 & 20,02 & 19,93 & 19,56 \\
\hline Módia & 21,28 & 19,66 & 20,17 & 19,56 & & \\
\hline
\end{tabular}

Quadro XIV: Análise da variância das médias do diâmetro externo das fibras nos diferentes níveis. (Planta B).

\begin{tabular}{|l|c|c|c|c|}
\hline & G.L. & S.Q & Q.M. & F \\
\cline { 2 - 5 } Anéis & 15 & 0,07934 & 0,00529 & 0,849 \\
Alturas & 3 & 0,17556 & 0,05852 & $9,393^{* *}$ \\
Residuo & 45 & 0,28034 & 0,00623 & \\
\hline
\end{tabular}

Não há significância para os aneis.

As alturas diferem significativamente ao nível de $1 \%$ de probabilidade. 
CIG. 1 - Esquema representativo em C.L. dos anéis de crescimento do tronco de uma
árvore de Eucalyptus saligna (planta A)

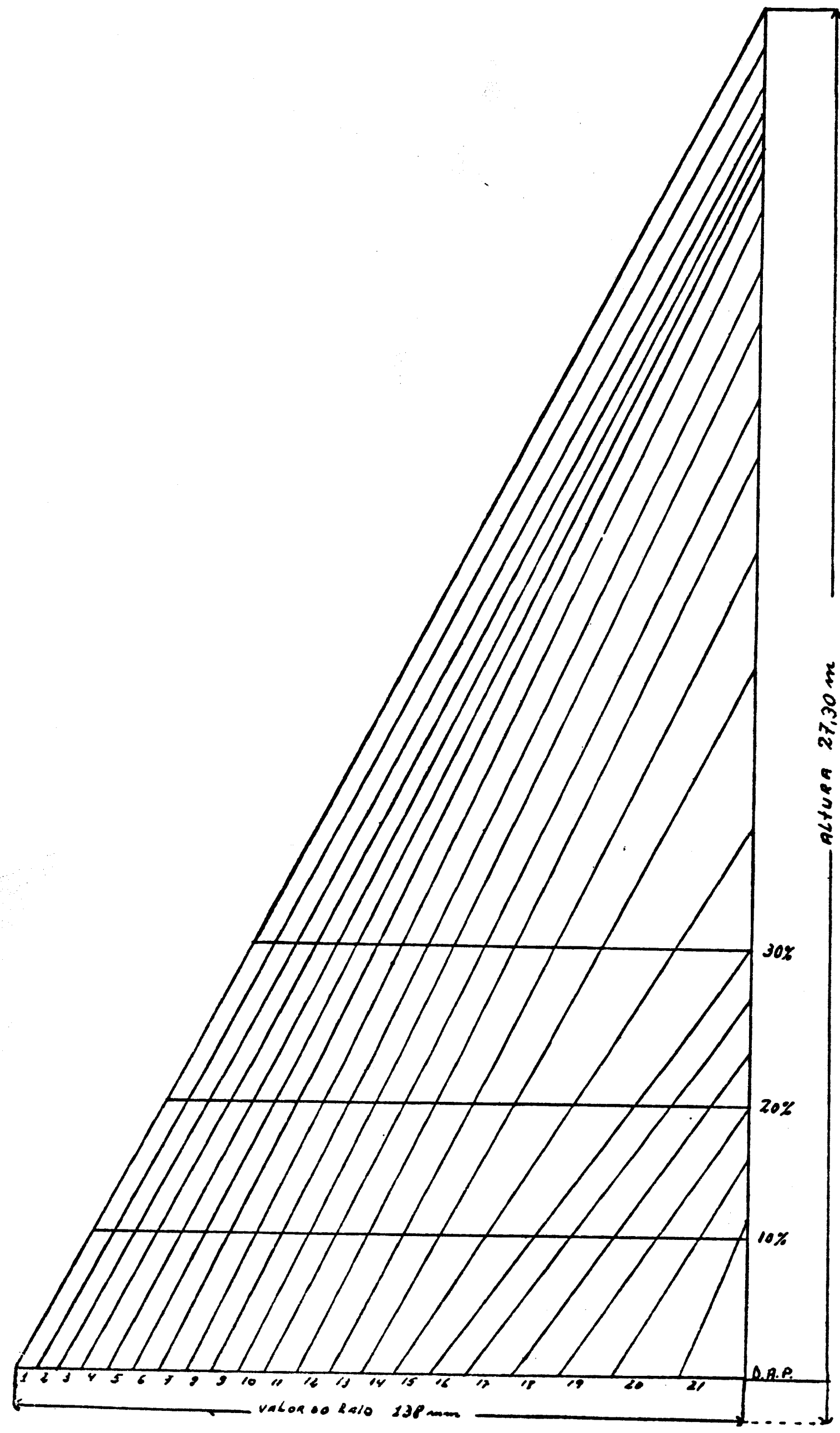




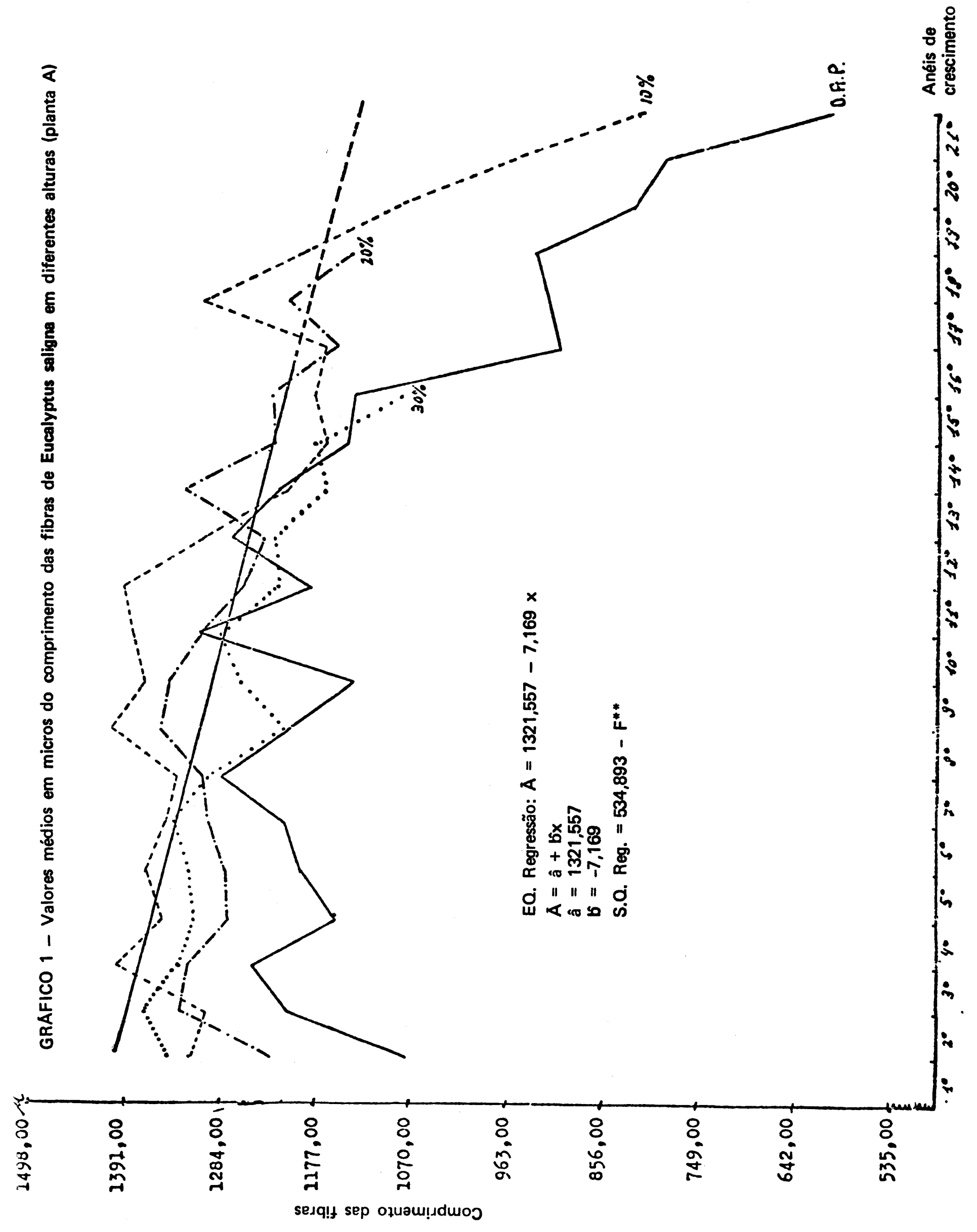




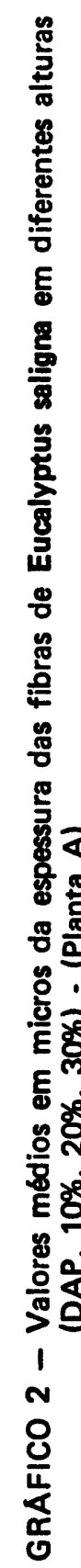

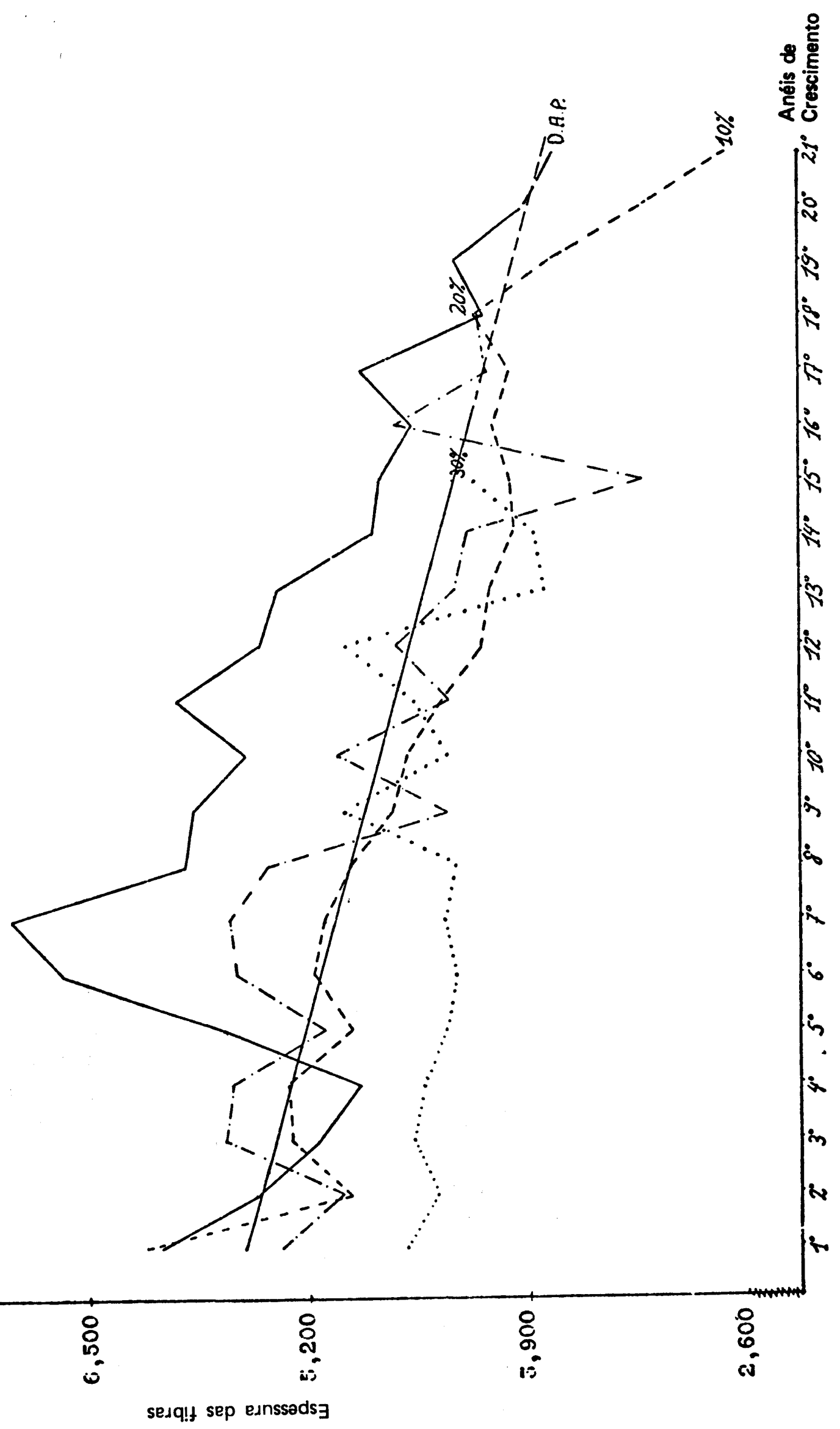




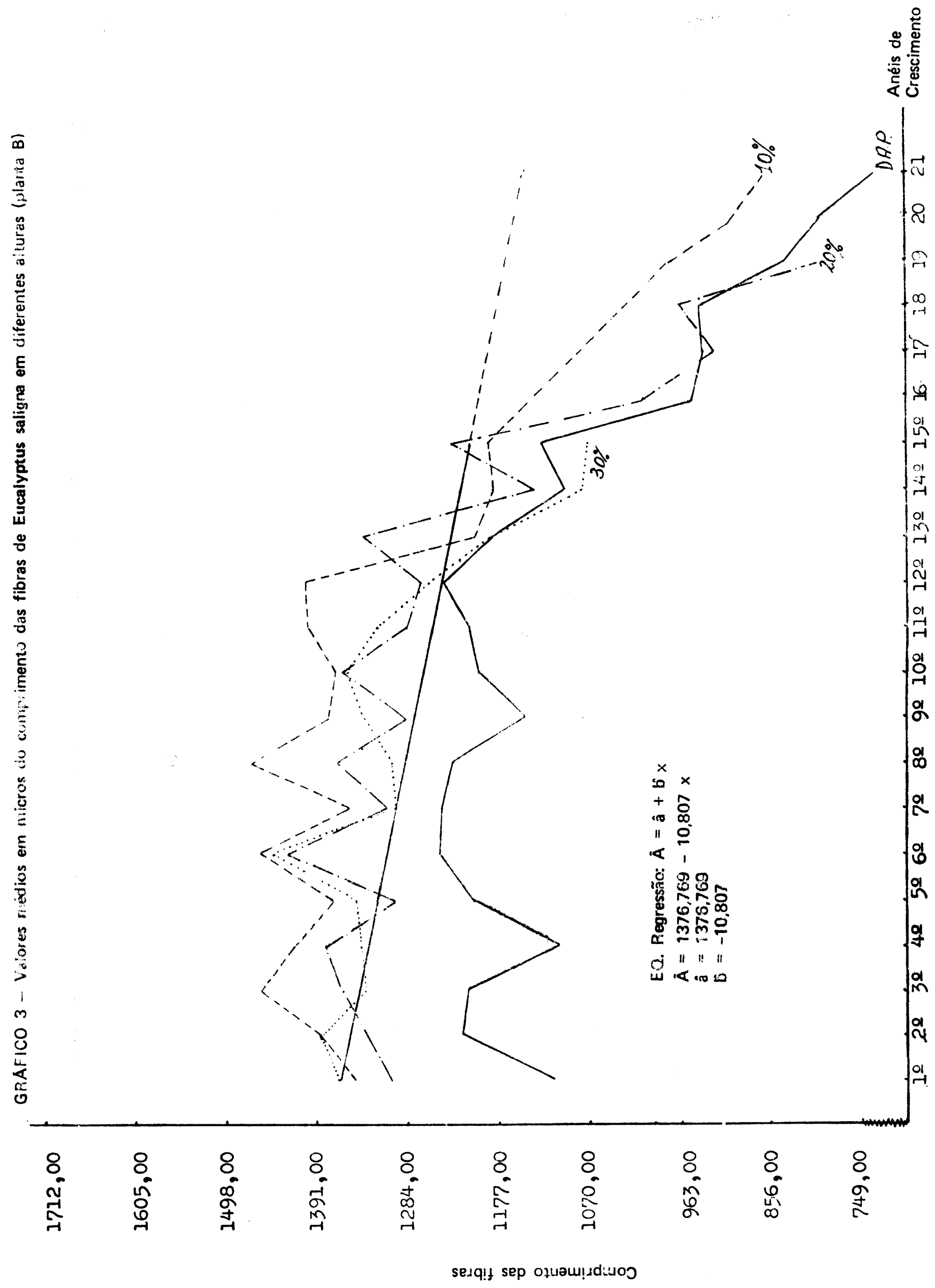




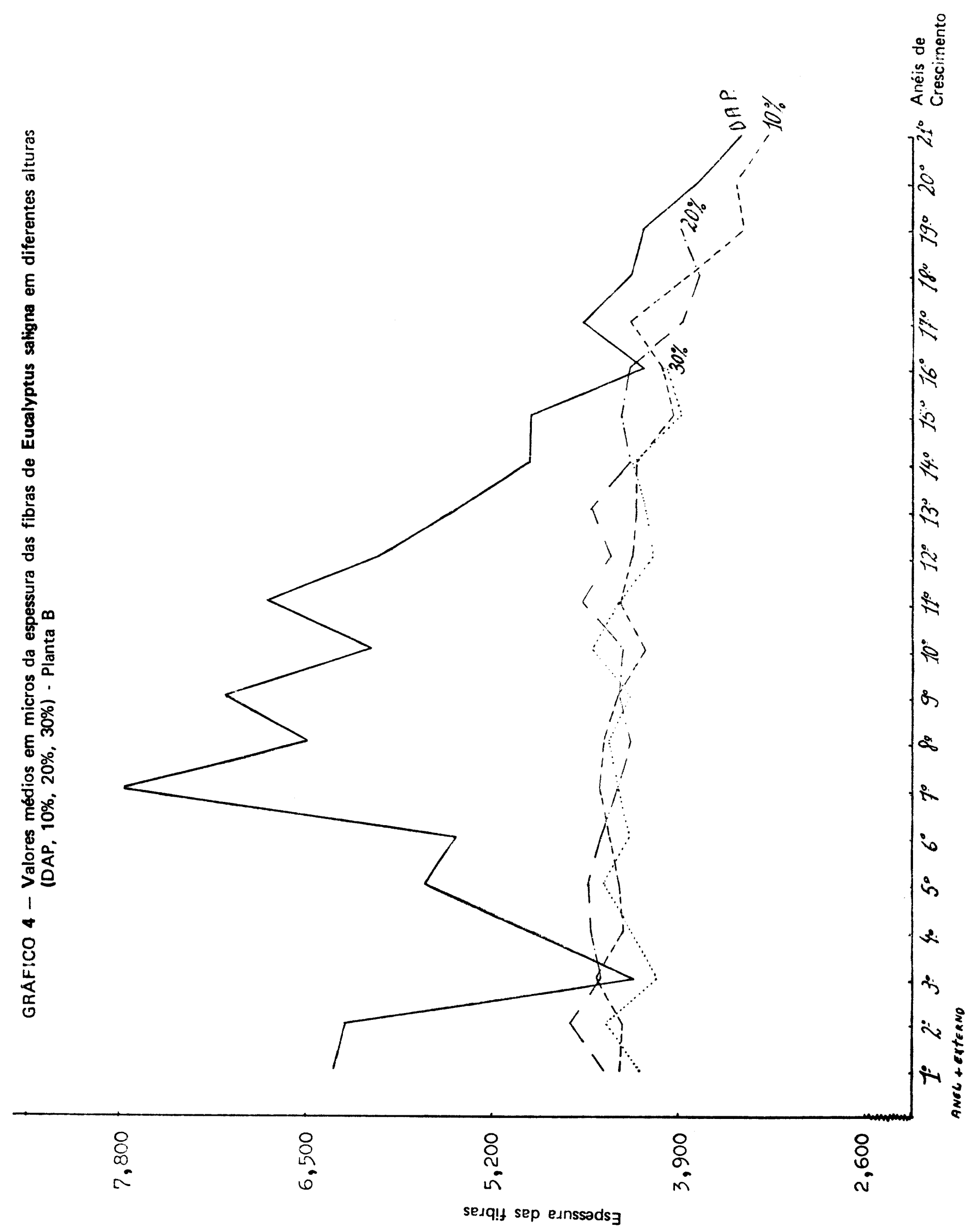


